\title{
A CASE OF GUSTATORY SWEATING
}

\author{
BY \\ H. I. TANKEL \\ From the Western Infirmary, Glasgow
}

Profuse facial sweating on eating is a rare phenomenon of interest not only as a clinical problem but also as a means of elucidating the complex mechanism of the nervous and hormonal control of perspiration. Of the cases described hitherto all except one have occurred as a result of sympathetic paralysis particularly in the distribution of the auriculo-temporal nerve. The present case is unusual in having no such predisposing cause and in presenting some unusual features on investigation.

The usual story is that an injury to the sympathetic supply to a part of the face is followed after an interval of anything from a few days to several months by sweating, of varying degree, on eating. Investigation confirms the deficient sympathetic supply and shows that certain drugs like acetylcholine and pilocarpine which normally cause sweating on injection produce an excessive response. The one case in which no sympathetic paralysis occurred was a patient with syringomyelia (Wilson, 1936).

A variety of explanations has been offered for the phenomenon of gustatory sweating. The sensitivity of the sweat glands to acetylcholine suggested that sweating might be due to a diffusion of this substance from the cholinergic nerve endings during reflex salivation (List and Peet, 1938). On the other hand it was suggested that since section of the sympathetic nerves had taken place there might have been abberrant regeneration in which other cholinergic fibres, say salivary fibres, came to supply sweat glands so that there would be a sweating response to any stimulus to salivation (Ford, 1933). Yet another suggestion was that there might be a double innervation to the sweat glands of the face, since sweating, which could be inhibited by peripheral nerve block, was seen to occur in an area where the sympathetic nerves were known to have degenerated (Wilson, 1936 ; Guttmann, 1931). Unfortunately none of these explanations can be applied to the present case in which, as will be shown, sweating appears to occur in response to stimulation through normal channels.

\section{The Physiology of Sweating}

The present view on the physiology of sweating (Kuno, 1934) is that generalized sweating is a response to stimulation of centres in the brain and spinal cord by emotional stimuli from the higher centres or by a rise of internal temperature due to exercise or warm surroundings. The fibres supplying the sweat glands of the face are anatomically sympathetic fibres which are nevertheless cholinergic (Dale and Feldberg, 1934). They arise in these centres, are relayed in the lateral horns of the first and second thoracic segments of the cord. and travel up the cervical sympathetic chain to be relayed again in the superior cervical ganglion. From there postganglionic fibres go to the plexus of nerves which surrounds the carotid arteries. The fibres from the internal carotid plexus join the ophthalmic division of the trigeminal nerve intracranially while those of the external carotid plexus are given off at various levels and join the second and third divisions of the trigeminal nerve extracranially. The sweat fibres are thus distributed with the terminal branches of the trigeminal nerve. No significant number, if any, is carried in the facial nerve (List and Peet. 1938) and none at all in the terminal branches of the arteries to the face (Wilson, 1936). Sirre the sweat glands are innervated by cholinergic fibres it is natural that acetylcholine and parasympathomimetic drugs such as mecholyl should produce sweating on subcutaneous injection. Pilocarpine also stimulates sweating by a direct action on the sweat glands themselves (Wilson, 1934). Histamine does not produce sweating unless an abnormally high dose or an intravenous injection gives rise to such severe side effects as to cause central stimulation (Weiss, Robb, and Ellis, 1932). The new and fascinating feature in the present case is that histamine produced an abnormal and localized sweating response in small 
dosage and so raised some interesting points as to its mode and place of action.

\section{Case Report}

P. M., a man aged 44, was admitted to the Western Infirmary, Glasgow, in April, 1949, for gastrectomy, following a history of duodenal ulceration since 1922.

Incidental to his peptic ulcer history, he complained of sweating on the left side of the face on eating. Sweating began almost immediately and became profuse in five minutes, remained so until five or ten minutes after the conclusion of the meal and then gradually subsided in the course of the next two to four hours. The sweating was greatest over the forehead but was also present around the eye, on the side of the nose, on the cheek, upper lip and chin, and extended backwards over the parietal region. Sweating stopped sharply in the midline of the face (Fig. 1).

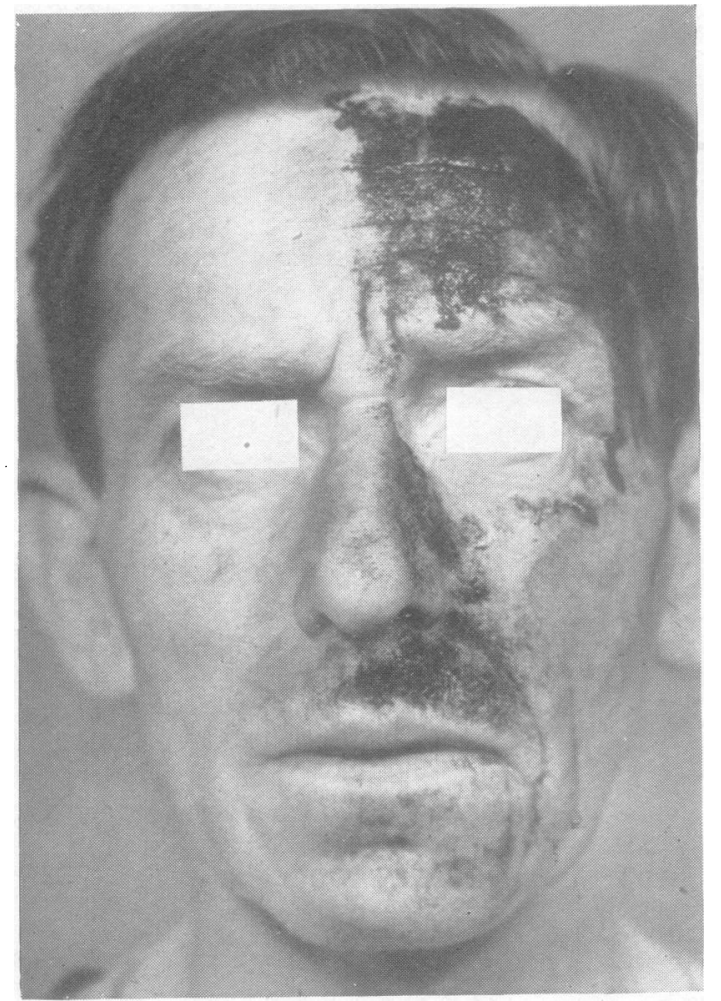

Fig. 1.-Area of gustatory sweating demonstrated by quinazerin.

The complaint was first noticed in 1946 and gradually increased in severity up to the present time when two or three handkerchiefs are required to mop the face at each meal. There was no history of injury or disease locally or referable to the central nervous system, but for the 10 or 12 years before the onset of the sweating the patient had suffered in the summer of each year from a sensitivity to sunlight which produced vesicula- tion on the forehead sometimes severe enough to keep him indoors. The patient is not certain whether only one side was affected, but this reaction to sunlight has not reappeared since the onset of the gustatory sweating.

On examination slight spontaneous sweating on the left side of the face was noted, especially in warm weather or following slight exertion.

There was no Horner's syndrome, the pupils were equal, and no abnormality of the central nervous system was detected. Skin temperature was taken by means - of a thermocouple and was identical on both sides. The right side was used as a control in this respect throughout the observations.

A radiograph of the skull and cervical spines showed calcification of the pineal gland, choroid plexuses, and posterior clino-petrous ligaments.

The Wassermann reaction was negative.

\section{Experiments}

Investigation of the Afferent Pathway.-In the previous work on the subject there has been no important difference of opinion as to the afferent pathway of the gustatory reflex arc. In the present case it was confirmed that sweating occurred in response to stimulation of taste fibres by food and that the response was proportional to the intensity of the stimulus. The chewing of tasteless cotton wool was ineffective, showing that salivation could occur without sweating and that the exteroceptive and proprioceptive fibres of the trigeminal nerve played no part. The application of cocaine to the tongue and mouth was inconclusive as there always seem to be taste fibres of the glossopharyngeal nerves which remain unanaesthetised (Fridberg, 1931). Reflexeso from the stomach were excluded, since $40 \mathrm{ml}$. of $7 \%$ ethyl alcohol, which is sufficient to stimulate the secretion of gastric juice, produced no response.

Cases have been described where the thought of food produced gustatory sweating showing that an afferent path from the higher centres might exist (Rappoport, 1920, cited by Fridberg), but this was not found to be so in the present case. The pathway is therefore considered to be by way of the taste fibres of the glossopharyngeal nerve and the chorda tympani component of the facial nerve (List and Peet, 1938).

Investigation of the Effector Mechanism.-As a first step in the investigation of the effector mechanism it was decided to try the effect of blocking the sympathetic nerve supply to the sweat glands of the face in various ways.

Effect of Supra-Orbital Nerve Block.-The left supraorbital nerve was blocked at the supra-orbital notch by the injection of about $2 \mathrm{ml}$. of $1 \%$ procaine hydrochloride without adrenaline. An area of anaesthesia was produced in the distribution of the nerve and spontaneous sweating stopped in the anaesthetic area. No obvious flush was noticed in this area. Gustatory sweating tested after five minutes was found to be completely inhibited in the anaesthetic area. This indicates that gustatory sweating is a reflex via fibres present in the supra-orbital nerve and not the result of direct pharmacodynamic action on the sweat glands. 
Effect of Cervical Sympathetic Block.-The left cervical sympathetic chain was blocked at the root of the neck by the injection of $20 \mathrm{ml}$. of $1 \%$ adrenaline free procaine hydrochloride. Spontaneous sweating stopped over the left half of the face and there was flushing of the area with a rise of skin temperature of $1 \cdot 3^{\circ} \mathrm{C}$. Gustatory sweating was tested after five minutes and there was no response. After 15 minutes minimal sweating reappeared on the left side presumably as the effect of the block wore off. This suggests that the fibres subserving the gustatory reflex arc are present in the cervical sympathetic chain and are therefore presumably sympathetic fibres.

Action of Atropine.-Atropine sulphate, gr. 1/100, was given subcutaneously. After 30 minutes the patient felt his mouth dry and had difficulty in accommodation. Spontaneous sweating stopped and the face became perfectly dry. Skin temperatures remained equal on both sides. Gustatory sweating was abolished as expected, since the nerves to the sweat glands are cholinergic and atropine acts by preventing the utilization of acetylcholine. Gustatory sweating therefore depends on the integrity of fibres present in the supra-orbital nerve and the cervical sympathetic chain, and is inhibited by atropine. There is no reason to suggest that these fibres are any other than the normal sweat fibres.

Action of Tetra-ethyl Ammonium Bromide.-Since tetra-ethyl ammonium bromide produces a block of preganglionic impulses at the sympathetic ganglia (Acheson and Moe, 1946) and so inhibits normal sweating it seemed reasonable to try its effect.

Tetra-ethyl ammonium bromide $0.5 \mathrm{~g}$. in $5 \mathrm{ml}$. was given slowly intravenously. The patient complained of a metallic taste in the mouth, generalized flushing, tingling of the extremities, and slight headache. Spontaneous sweating stopped. After five minutes a gustatory stimulus was applied and in a further three minutes sweating became obvious over the gustatory sweating area and was of moderate degree. All subjective effects of the tetra-ethyl ammonium bromide disappeared after 18 minutes and sweating continued on the left side for about one hour. The gustatory sweating reflex was therefore not inhibited completely by the action of tetra-ethyl ammonium bromide. The reason for this is uncertain.

Demonstration of Other Sweating Abnormality.-The next step in the investigation was to see if any other abnormality of sweating could be demonstrated in the gustatory sweating area and so the effect of heat, parasympathomimetic drugs, and pilocarpine was observed.

Effect of Heat.--The patient was stripped, covered in a blanket, and placed in a heat cage with the face showing but protected from direct heat. Sweating began on the trunk and on the affected left side of the face after about six minutes. After 11 minutes sweating appeared on the right forehead and after 35 minutes was profuse over the entire body. The left side of the face always sweated more than the right side, even after a further half-hour of drenching sweat. There was thus an excessive response to heat on the affected side. It is interesting that a similar excessive response to heat was found in the patient with syringomyelia mentioned at the beginning of the article.

Action of Acetylcholine.-Acetylcholine, mg. 50 injected subcutaneously, produced slight sweating on the left side of the face noticeable in eight minutes and lasting for five to ten minutes. A substantially similar result was obtained with the injection of mg. 5 of mecholyl.

Action of Pilocarpine.-Pilocarpine nitrate, 1/12 gr., was injected subcutaneously. Sweating became noticeable in three minutes and was profuse in 15 minutes, lasting for more than an hour. The sweating was most marked on the left side of the face, being comparatively slight on the right side. There did not appear to be any flush, and skin temperatures remained equal on both sides.

The left side of the face appears to be more sensitive to the action of heat, acetylcholine, and pilocarpine than the right side. Whether it is the glands themselves or another part of the gustatory sweating reflex arc which are sensitive remains to be decided.

Since a sensitivity of some sort appeared to be present it was decided on empirical grounds to try the effect of an injection of histamine. This had interesting results.

Action of Histamine.-Histamine acid phosphate, mg. 0.5, was injected subcutaneously. Definite sweating of moderate degree and involving only the left side of the face appeared within seven minutes and remained for 27 minutes. There was no accompanying malaise and skin temperatures remained equal on both sides. As has already been mentioned histamine does not produce sweating except as a result of severe side effects from overdosage. In such a case sweating is generalized. Here sweating was definitely localized to the gustatory sweating area.

The action of histamine was found to be completely abolished by an injection of atropine, and by supraorbital nerve block, and cervical sympathetic block. These facts, when taken in conjunction with the localization of its action, suggest that histamine plays a part in the gustatory reflex arc. If so, it must act by way of the normal sweat fibres to the face since its action is inhibited when they are blocked.

Effect of Antihistamine Drugs on Gustatory Sweating. -The effect of the antihistamine drugs on gustatory and heat sweating was next investigated.

In order that approximate comparisons of effect could be made the drugs were used in therapeutic doses. The parenteral route was chosen, though not usually the route of choice, so as to avoid the possibility of an unwanted gustatory response.

'Anthesan ', 50 mg., was given by deep subcutaneous injection. After 15 minutes all spontaneous sweating stopped and a stimulus by eating was applied, but only very slight sweating over the left half of the face resulted showing that the gustatory sweating response was greatly inhibited, and it remained so for about two and a half hours. 
When the observation was repeated with 'antistin', mg. 50, no appreciable effect on gustatory sweating was noted.

'Benadryl 'was found to have an action intermediate between that of ' anthesan' and ' antistin'.

The effect of these drugs in inhibiting gustatory sweating was thus in proportion to their relative antihistamine activity, ' anthesan' having the greatest antihistamine effect and ' antistin' the least (Graham, 1947). Spontaneous sweating probably stopped on account of the slight anti-acetylcholine effect of these drugs where ' antistin' has a greater effect than 'anthesan'.

Effect of Antihistamine Drugs on Heat Sweating. - The drugs were given in the same dose and by the same route as above and heat sweating was carried out under the same conditions as described before.

'Anthesan' was given, and after 15 minutes when spontaneous sweating had stopped, the heat cage was applied. The trunk became moist in 10 minutes but the face remained perfectly dry until, after $\mathbf{4 0}$ minutes, the left side began to sweat, followed by the right side seven minutes later. Sweating on the right side then overtook the left side in intensity and after 68 minutes sweating on the right side was more profuse than on the left.

When 'antistin' was given the normal response to heat sweating was substantially unaffected. The left side sweated earlier than the right side and always more profusely.

Once again the action of 'benadryl' was intermediate between that of 'anthesan' and 'antistin', sweating being almost equal on both sides at the end of an hour.

When the dose of ' benadryl' was doubled, sweating on the left side was further inhibited so that for the first time sweating began on the right side and remained more profuse on that side throughout the test. These drugs therefore acted in proportion to their antihistamine effect on heat sweating but only produced an appreciable effect in the gustatory sweating area. The anti-acetylcholine effect of these drugs was insufficient to influence the right side to any appreciable extent and so may be considered to have had a negligible effect on the left side. The contention therefore is that the inhibition of heat sweating on that side may be considered as almost entirely due to antihistamine activity. The corollary to this proposition is that the greater part of the sweating which appears on the left half of the face in response to heat is in fact due to histamine activity.

\section{Discussion}

As has already been pointed out, in a normal individual histamine does not produce sweating unless as a result of side effects. Sweating cannot be produced in a normal or sympathectomized area of skin by local injection, nor does a normal or sympathectomized limb sweat when histamine is injected intra-arterially.

The notable features in this case are that histamine produced sweating at all and that this sweating was produced only in the gustatory sweating area.
The accurate localization of the sweating suggests that histamine or a similar substance plays a part in the gustatory sweating reflex arc. A further feature in support. of this argument is the finding that the antihistamine drugs inhibit gustatory sweating in proportion to their antihistamine activity.

A further point of interest is the inhibition of heat sweating, a sympathetic but cholinergic activity, by the antihistamine drugs. These drugs do have a slight anti-acetylcholine effect which has already been shown to be negligible: It is therefore argued that the inhibition of heat sweating on the left side of the face by the antihistamine drugs might be considered as almost entirely due to their antihistamine effect and that the explanation of the excessive response to heat on that side is that it is due to histamine activity.

Where and how does this histamine act? One hypothesis might run as follows (Wilson, 1950). The stimuli to generalized sweating are emotion or a rise in blood temperature acting on a centre in the brain. If a part of this centre were in a state of constant vasodilatation it might respond readily to stimuli which did not affect other parts of the centre. If this vasodilatation were reduced or abolished by antihistaminics the sweating response to heat and other forms of central stimulation in the corres ponding skin areas would be diminished, as in face they were. An injection of histamine would for short time increase the vasodilatation and so mighe? increase the sensitivity of the centre to subliminal stimuli, producing sweating in the affected region.

The mechanism of the transmission of afferent nerve impulses in the central nervous system is unknown, but since some nerves have been shown to yield moderate quantities of histamine on extraction(Von Euler, 1948), they may be histaminergic that is to say, producing histamine at their nerve endings. If the afferent taste fibres of the salivary reflex were histaminergic, then following very strong stimuli, there would be an overflow of histamine which would make the neighbouring sweat centres hyperactive. This might explain the phenomenon of normal facial sweating on eating very spicy foods. Abnormal gustatory sweating as described would then be explained by constant excessive production of histamine with chronic vasodilatation in a strictly localized part of the centre. No cause has been suggested, nor has any explanation been given as to how it is so strictly localized. It is also difficult to fit into this picture the failure of tetraethyl ammonium bromide to inhibit gustatory sweating.

On the other hand, suppose that due to an abnormality in the efferent fibres they became 
sensitive to histamine. Since the action of histamine is abolished by cervical sympathetic block the abnormality would have to be in the preganglionic fibres or central to them. If the abnormality of the fibres was such that an abnormal substance was formed at the nerve ndings when an impulse passed through them, then if this substance were - histamine or similar to it and under these conditions produced an excessive response, the excessive sweating response to all forms of central stimulation would be explained.

If subliminal sweat impulses normally occur in response to food then gustatory sweating would be explained as occurring in response to the reinforcement of these normal impulses by the histamine-like substance.

The failure of tetra-ethyl ammonium bromide to inhibit gustatory sweating might then be explained as a failure to block an abnormal chemotransmitter, the histamine-like substance produced by the abnormal pre-ganglionic fibres. The effect of tetra-ethyl ammonium bromide on histamine sweating was not tried as it was felt that there was some risk to the patient involved in the fall of blood pressure.

The antihistamine drugs would inhibit all forms of sweating since they would inhibit this histaminelike chemotransmitter.

The pathology of this abnormality is again unknown. Perhaps some form of neuritis in the preganglionic sweat fibres would explain it. A similar neuritis in the patient with syringomyelia mentioned before would explain the similarity in the reactions to heat, acetylcholine, and pilocarpine in the two cases. In that case it might be explained as a response to the pressure produced by the cavitation of the cord peculiar to the disease. However, neuritis of various types usually results in a loss of function of the nerve rather than an increase, and the whole hypothesis is rendered even more unsatisfactory by the lack of evidence for the occurrence of histamine in efferent sympathetic fibres, or of its production by them.

It has not been forgotten that the afferent side of the reflex arc might be responsible for the syndrome but it is very difficult to test this assumption.

There are obviously great gaps in the reasoning of the explanations offered but they are intended to indicate a tentative line of thought and not to be cast-iron deductions. It is difficult in any other way to find an explanation for the actions of histamine and the antihistamine drugs which have been described.

I wish to express my thanks to Prof. C. F. W. Illingworth for the helpful advice and encouragement which he has given me, and to Prof. W. C. Wilson for his valuable criticisms and suggestions.

\section{REFERENCES}

Acheson, G. H., and Moe, G. K. (1946). J. Pharmacol., 87, 220.

Dale, H. H., and Feldberg, W. (1934). J. Physiol., Lond., 82, 121.

Ford, F. R. (1933). Arch. Neurol. Psychiat., Chicago, 29, 1279.

Fridberg, D. (1931). Dtsch. Z. Nervenheilk., 121, 225.

Graham, J. D. P. (1947). J. Pharmacol., 91, 103.

Guttmann, L. (1931). Z. ges. Neurol. Psychiat., 135, 1.

Kuno, Y. (1934). "The Physiology of Human Perspiration." London.

List, C. F., and Peet, M. M. (1938). Arch. Neurol. Psychiat., Chicago, 40, 443.

Rappoport, M. (1920). Cited by Fridberg.

Von Euler (1948). J. Physiol., Lond., 107, 10 P.

Weiss, S., Robb, G. P., and Ellis, L. B. (1932). Arch. intern. Med., 49, 360.

Wilson, W. C. (1934). Brain, 57, 422.

-(1936). Clin. Sci., 2, 273.

(1950). Personal communication. 